\title{
The interaction between organic phosphate ester and p53: An integrated experimental and in silico approach
}

\author{
Fei Li ${ }^{\mathrm{a}, \mathrm{b}, *}$, Renmin $\mathrm{Li}^{\mathrm{c}}$, Xianhai Yang ${ }^{\mathrm{d}}$, Liping You ${ }^{\mathrm{a}, \mathrm{b}}$, Jianmin Zhao ${ }^{\mathrm{a}, \mathrm{b}}$, Huifeng $\mathrm{Wu}^{\mathrm{a}, \mathrm{b}, *}$

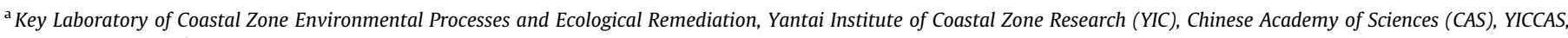 \\ Yantai 264003, PR China \\ ${ }^{\mathrm{b}}$ Shandong Provincial Key Laboratory of Coastal Zone Environmental Processes, YICCAS, Yantai 264003, PR China \\ ' Qingdao Institute of Bioenergy and Bioprocess Technology, Chinese Academy of Sciences, Qingdao 266101, PR China

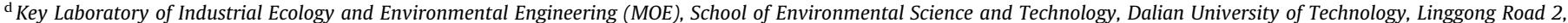 \\ Dalian 116024, PR China
}

\section{A R T I C L E I N F O}

\section{Article history:}

Available online 8 January 2014

\section{Keywords:}

Organophosphate flame retardants (OPFRs) p53

Docking

Quantitative structure-activity relationship (QSAR)

Binding affinity

Multiple linear regression (MLR)

\begin{abstract}
A B S T R A C T
Concerns have been raised in regards to the environmental impact of the more used organophosphate flame retardants (OPFRs). In this study, to better understand the relationship between molecular structural features of OPFRs and binding affinity for the tumor suppressor p53, an integrated experimental and in silico approach was used. From docking analysis, hydrogen bonding and hydrophobic interactions were found to be the dominant interactions, which implied the binding affinities of the compounds. The binding constants of 5 OPFRs were determined by surface plasmon resonance technology (SPR). Based on the observed interactions, appropriate molecular structural parameters were adopted to develop a quantitative structure-activity relationship (QSAR) model. The developed QSAR model had good robustness, predictive ability and mechanism interpretability. The interactions between the OPFRs and p53 $\left(E_{\text {binding }}\right)$ and the partition ability of the OPFRs into the bio-phase are main factors governing the binding affinities.
\end{abstract} (c) 2013 Elsevier Ltd. All rights reserved.

\section{Introduction}

Owing to the phase-out of the commercial penta-BDE mixture in 2004, an increasing number of organophosphate flame retardants (OPFRs) are being used around the world and are consequently being introduced into the environment (Dishaw et al., 2011). Since OPFRs are not covalently bound to host materials, these compounds could diffuse out into surroundings relatively easily by volatilization, leaching or abrasion (Marklund et al., 2003; Reemtsma et al., 2008). OPFRs are widely distributed owing to their extensive use, and volatility, and are considered to be persistent in water and air (Reemtsma et al., 2008).

The chlorinated alkylphosphates such as tris-(2-chloro-, 1methyl-ethyl)-phosphate (TCPP) and tris-(2-chloroethyl)-phosphate (TCEP) are mostly used as flame retardants in polyurethane foam (Andresen et al., 2004). The non-derivatised alkylphosphates such as tri- $n$-butylphosphate (TnBP), tri-iso-butylphosphate (TiBP), triphenylphosphate (TPP) and tris-(butoxyethyl)-phosphate (TBEP) are predominantly utilised as plasticisers, lubricants and to

\footnotetext{
* Corresponding authors at: Key Laboratory of Coastal Zone Environmental Processes and Ecological Remediation, Yantai Institute of Coastal Zone Research (YIC), Chinese Academy of Sciences (CAS), YICCAS, Yantai 264003, PR China. Tel.: +86535 2109189; fax: +865352109000 (F. Li). Tel.: +86535 2109190; fax: +86535 2109000 (H. Wu)

E-mail addresses: fli@yic.ac.cn (F. Li), hfwu@yic.ac.cn (H. Wu).
}

regulated pore sizes, e.g., in concrete, though in some cases, they are also used as flame retardants. Many of these flame retardants and plasticizers have been frequently detected in various environments, including indoor and outdoor air (Hartmann et al., 2004), indoor dust (Marklund et al., 2003; Stapleton et al., 2009; Takigami et al., 2009), water (Bacaloni et al., 2008; Regnery and Puttmann, 2010), sediments (Garcia-Lopez et al., 2009), soils (David and Seiber, 1999), landfill leachates (Yasuhara et al., 1999) and even in the aquatic organisms and in human breast milk (Liu et al., 2012).

OPFRs are structurally similar to neurotoxic organophosphate pesticides, raising concerns about exposure and toxicity to humans (Dishaw et al., 2011). Previous studies have demonstrated that some OPFRs, especially chlorinated OPFRs, are highly persistent in the environment (Meyer and Bester, 2004; Regnery and Puttmann, 2010), and they can even persist in drinking water after conventional treatment, indicating that these compounds can reach the human body via drinking water (Stackelberg et al., 2004; Andresen and Bester, 2006). However, little is known about the toxicity of OPFRs, although some studies indicate that TPP and TnBP are suspected to be neurotoxic (Martinez-Carballo et al., 2007), while others like TCEP, TDCP and TCPP are carcinogenic for animals (Matthews et al., 1990; Commission, 2001).

TPP has been shown to be a potent human blood monocyte carboxylesterase inhibitor (Matthews et al., 1990; Commission, 
2001). Considering their toxicity, much more attention should be paid to human exposure and the potential human health risk (Matthews et al., 1990; Commission, 2001). OPFRs can interact with DNA, which may cause DNA damage, mutation and induced cancer. Therefore, it is of great importance to study the mechanism of gene damage caused by OPFRs and their structure-activity relationship for the interaction.

Optical biosensors based on surface plasmon resonance technology (SPR) are a dynamic tool for biomedical and pharmaceutical research (Sipova and Homola, 2013). These biosensor-based assays measure the competition between the interactions of a specific biological recognition element with the target analyte (e.g., toxin) immobilized onto the sensor chip surface and in the sample (Campbell et al., 2009). SPR biosensors have been applied to the study of a very diverse set of interaction partners including receptors, antibodies, antigens, enzymes, growth factors, glycoproteins, nucleic acids, drugs, membranes, cells and viruses (Karlsson, 2004).

Given the large number of compounds that may bind to the receptors, there is increasing interest in developing computational methods (in silico) to predict affinity of compounds with the receptors, including quantitative structure-activity relationships (QSARs). Furthermore, molecular docking have become an integral part of many modern structure-based computational simulations of chemicals (Martinez et al., 2008). Docking methodologies use the knowledge of three-dimensional structure of a receptor in an attempt to optimize the bound ligand or a series of molecules into the active site. Combinational use of docking with QSAR can provide more information on the interaction between the ligand and the receptor (Li et al., 2010a,b, 2012).

In this study, the tumor suppressor p53 was used for target function, the binding constants for OPFRs interacting with p53 were determined. Combined with molecular simulation and in vitro test, the mechanism of OPFRs interacting with p53 was studied. From docking analysis, the interaction between OPFRs and p53 were established. Finally, the theory prediction model for the binding constants of OPFRs interacting with p53 was developed. The results could be conducive to understand the genetic mechanism for OPFRs compounds, and could provide the theory basis for their pollution prevention and control.

\section{Materials and methods}

\subsection{Total RNA extraction}

Total RNA was extracted from zebra fish using the TRIzol Reagent (Invitrogen, USA) following the manufacturer's protocol. The extracted RNA was then treated with RQ1 RNase-Free DNase (Promega, USA) to remove DNA contamination.

\subsection{Cloning of the full-length cDNAs for p53}

Tumor suppressor protein p53 cDNA fragments were amplified and cloned from the total RNAs by reverse transcription and polymerase chain reaction method (RT-PCR) using p53-specific primers 5'-GACTATCCCGGCGATCATGGATT- $3^{\prime}$ and $5^{\prime}$ - TTTCTTGAAGTTG CTCTCCTCAG-3'. Single-stranded CDNAs were synthesized from one microgram of the total RNA using M-MLV reverse transcriptase (Promega, USA) according to the manufacturer's protocol.

\subsection{Induction and expression for $p 53$}

The final amplified product was cloned in the pET28a expression vector (Novagen). The recombinant plasmids were prepared by an alkaline lysis procedure and sequenced to confirm the size and orientation of the insert. The core DNA-binding domain was overproduced in Escherichia coli BL21 (DE3). The cells were incubated at $37{ }^{\circ} \mathrm{C}$ until an OD of $0.5-0.8$ had been attained. Then, $0.6 \mathrm{mM}$ isopropyl $\beta$-D-thiogalactoside was added to induce the expression of the recombinant protein, and the incubation was continued at $25^{\circ} \mathrm{C}$ for $6 \mathrm{~h}$. All subsequent procedures were performed at $4{ }^{\circ} \mathrm{C}$. The cells were harvested by centrifugation and were washed and purified as previously described (Xue et al., 2009). Then, the purified p53DBD was checked on an SDS-polyacrylamide gel for purity.

\subsection{Binding constants for OPFRs using biacore analyses}

The interaction between p53 protein and OPFRs was studied by surface plasmon resonance (SPR) on Biacore T100 (GE Healthcare) using CM5 sensor chip at $25^{\circ} \mathrm{C}$. Research grade CM5 sensor chips were employed. The CM5 sensor chip was activated using the recommended protocol (BIAapplications Handbook, BIAcore, Uppsala, Sweden). The zebra fish p53 DBD was immobilized on a CM5 chip. The immobilization levels were selected for binding and kinetic assays, respectively. The running buffer was filtered (pore size $0.22 \mathrm{mM}$ ) and degassed using a filtration apparatus immediately before use.

The CM5 sensor chip surface was activated by injection of EDC/ NHS (1:1) at $10 \mu \mathrm{L} / \mathrm{min}$ for $7 \mathrm{~min}$, then injection of $50 \mu \mathrm{g} / \mathrm{mL}$ p53 in $10 \mathrm{mM}$ sodium acetate $\mathrm{pH} 5.0$ at $10 \mu \mathrm{L} / \mathrm{min}$ for $7 \mathrm{~min}$ to immolize p53 on the CM5 sensor chip surface, deactivate excessive reactive groups using ethanolamine. The binding of ligands to the p53 immobilized on the sensor chip was monitored in real time, using HBS with $0.05 \%$ P20 and 5\% DMSO as the running buffer $(10 \mathrm{mM}$ HEPES, $0.15 \mathrm{M} \mathrm{NaCl}, \mathrm{pH} 7.4$ ) at the flow rate of $30 \mathrm{ml} / \mathrm{min}$. The sensorgrams were fitted with a 1:1 binding model using BIA T100 evaluation software (GE Healthcare).

In the standard SPR analysis, the association rate constant $\left(k_{a}\right)$ and dissociation rate constant $\left(k_{d}\right)$ are obtained individually, and the dissociation constant $\left(K_{D}\right)$ value can be determined by the quotient of the rate constants $\left(k_{d} / k_{a}\right)$. More details could be found in the previous study (Drake et al., 2012; Katayama et al., 2012).

\subsection{Simulation for binding interaction by molecular docking}

Homologous 3D model of p53DBD was built on SWISS-MODEL net server. The binding interactions were studied by CDOCKER, which has been incorporated into Discovery Studio 2.5 (Accelrys Software Inc.) through the Dock Ligands protocol. CDOCKER is an implementation of the docking tool based on the CHARMm force field that has been proven to be viable (Wu et al., 2003). In CDOCKER, random ligand conformations are generated through molecular dynamics, and a variable number of rigid-body rotations/ translations are applied to each conformation to generate the initial ligand poses. The conformations are further refined by gridbased simulated annealing in the receptor active site, which makes the results accurate. From the docking analysis, insights into the interactions between the ligands and the receptor were gained. Additionally, the binding free energies ( $E_{\text {binding }}$ ) of the 5 OPFRs were obtained.

\subsection{Molecular structural descriptor generation and QSAR development}

It was hypothesized that the binding activity of OPFRs depended on the following two processes: (a) the penetration of OPFRs through bio-membrane and reaching the target site of action, and (b) the interactions between the OPFRs and p53. Thus, the logarithm of octanol/water partition coefficient $\left(\log K_{O W}\right)$ and $E_{\text {binding }}$ were selected to describe these processes and construct QSAR models. $\log K_{\text {OW }}$ was purposely selected to describe the partition process. $E_{\text {binding }}$ was also considered because binding to 
the receptor was the initiating step in toxicity by chemicals. $\log K_{O W}$ values were computed by the EPI Suite $4.0^{\mathrm{TM}}$ (http://www.epa.gov/ oppt/exposure). QSARs model were developed using MLR, an easily applicable and unambiguous algorithm suggested by OECD (2007). In MLR, multicollinearity among the input variables may result in wrong signs and magnitudes of regression coefficients. Thus the variance inflation factor (VIF) was adopted to evaluate multicollinearity among the input variables. VIF values exceeding 10 are often regarded as serious multicollinearity. The robustness of the model was evaluated by internal validation, and the leave-one-out crossvalidated coefficient $\left(Q_{L O O}^{2}\right)$ was calculated. The performance of external validation was characterized by the root mean square error (RMSE). These parameters are defined as follows (Schüürman et al., 2008):

$Q_{\mathrm{LOO}}^{2}=1-\sum_{i=1}^{n}\left(y_{i}-\hat{y}_{i}\right)^{2} / \sum_{i=1}^{n}\left(y_{i}-\bar{y}\right)^{2}$

$R M S E=\sqrt{\sum_{i=1}^{n}\left(y_{i}-\hat{y}_{i}\right)^{2} / n}$

where $y_{i}$ and $\hat{y}_{\mathrm{i}}$ are the observed and predicted values for the $i$-th compound, respectively. $\bar{y}$ is the average response value of the training set. $n$ stand for the number of compounds in the training sets.

\section{Results and discussion}

\subsection{Expression and purification of tumor suppressor protein $p 53 D B D$}

To investigate the binding reaction between p53DBD and OPFRs, a truncated form of p53DBD was expressed and purified. SDS-PAGE analysis showed that the $57 \mathrm{kDa}$ p53DBD protein was the predominant polypeptide in the purified fraction (Fig. 1). The concentration of p53DBD protein in this fraction was estimated to be $0.83 \mathrm{mg} / \mathrm{L}$.

\subsection{Binding kinetic analysis between p53 and OPFRs}

When OPFRs reacts with p53DBD to form complex, the complex formation depends on the free concentrations of OPFR, p53DBD and the stability of the complex, which can be described by the following equation:

$\frac{d[\mathrm{OPFR}-\mathrm{p} 53 \mathrm{DBD}]}{d t}=k_{a}[\mathrm{OPFRs}][\mathrm{p} 53 \mathrm{DBD}]-k_{d}[\mathrm{OPFR}-\mathrm{p} 53 \mathrm{DBD}]$

where $k_{a}$ is the association rate constant and $k_{d}$ is the dissociationrate constant. The p53DBD was immobilized on gold surface and

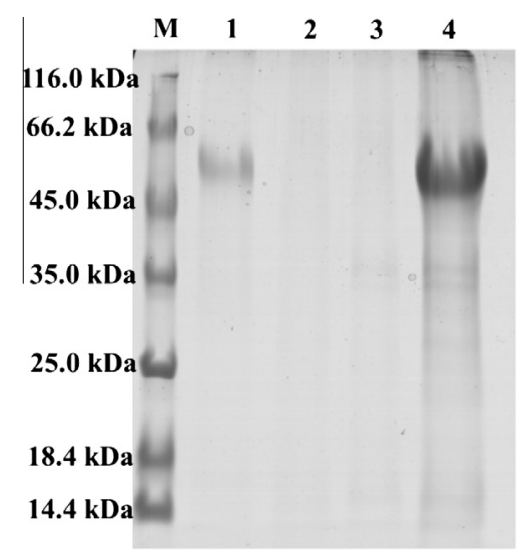

Fig. 1. The purified p53DBD analyzed by SDS-PAGE (sodium dodecyl sulfate-PAGE).
OPFRs was injected into the flow system as the analyte. The kinetic constants of the binding process were given in Table 1 .

\subsection{Docking analysis}

The docking view of three representative OPFRs (TCEP, TEP and TPrP) in the binding site of p53 was shown in Fig. 2. At the deep end of the pocket, His282 and Ala275 serve as anchoring points for the ligands, which explains the predominant contribution of the hydroxyl group to the binding affinity of chemicals. The ligands also interact with another polar region within the binding pocket, that is, with Val141.

As shown in Fig. 2, hydrogen bonding and hydrophobic interactions are characteristic interactions between the OPFRs and p53. As shown in Fig. 2, there are mainly three types of H-Bonds: (a) Hbonds formed between the oxygen of TCEP, TPrP and the hydrogen of Ala129 and His182, (b) H-bonds between the hydrogen of TCEP, TEP with the carbonyl oxygen of Val141, and (c) H-bonds between the chlorine of TCEP and phenyl hydrogen of His182 and Leu162. Acting as an 'anchor', the hydrogen-bonding intensely determines the 3D space position of the chemicals in the binding pocket, and facilitates the hydrophobic interaction of the OPFRs with the side chain of Val141, Arg181, Ile163, Ala129, His182, Ser183. Fig. 3 shows the electrostatic potential of the ligand binding site for p53. The binding site has positive potentials, from which it can also be concluded that the negative potentials of the OPFRs molecules facilitate them to bind with $\mathrm{p} 53$.

The binding free energies ( $E_{\text {binding }}$ ) of the 5 OPFRs are listed in Table 1. A simple linear free energy relationship (LFER) between $\log K_{D}$ and $E_{\text {binding }}$ was obtained (Fig. 4), which further proved that the binding to p53 was a key step for OPFRs to exert their toxicity. However $E_{\text {binding }}$ itself was not a good predictor for $\log K_{D}$, as indicated by the big prediction residuals for some OPFRs (Fig. 4). Thus, it is necessary to develop poly-parameter QSAR models for $\log K_{D}$ prediction.

\subsection{Development and validation of the QSAR model}

For the training compounds, MLR analysis with $\log K_{D}$ as the dependent variable and the selected parameters as predictor variables resulted the following optimal QSAR model:

$\log K_{D}=-5.41+5.53 \times 10^{-3} V+1.36 \times 10^{-2} E_{\text {binding }}$

$n=5, R^{2}=0.990, Q_{\mathrm{LOO}}^{2}=0.968, R M S E=0.078, p<0.01$

where $p$ is the significance level.

The VIF values of $V$ and $E_{\text {binding }}$ are 1.29 and 1.27, respectively. For the current QSAR model, all the predictor variables are statistically significant $(p<0.005)$, and all the VIF values are $<2$, implying that the QSAR model overcomes the problem of multicollinearity and thus the values of the regression parameters are genuine.

The $R^{2}$ value of the QSAR model is 0.990 , indicating a good goodness-of-fit of the model. $Q_{L O O}^{2}$ of the model is as high as 0.968 , implying good robustness of the model. The differences between $R^{2}$ and $Q_{L o O}^{2}$ do not exceed 0.3 , indicating no over-fitting in the model (42). The model has acceptable predictability, as indicated by RMSE $=0.078$.

In the developed model, the $t$ statistics for $V$ and $E_{\text {binding }}$ are 6.83 $(p<0.05)$ and $14.3(p<0.005)$, respectively, indicating that these parameters are statistically significant. $\log K_{D}$ values of OPFRs increase with $V$ values. The parameters molecular volume $(V)$ was selected to partly describe the partition ability of OPFRs. $V$ correlates with $\log K_{\text {OW }}$ positively, implying that OPFRs with large $V$ values tend to partition into the biophase easily. Moreover, the 
Table 1

The association rate constant $\left(k_{a}\right)$, the dissociationrate constant $\left(k_{d}\right)$, the binding constant $\left(K_{D}\right)$ and the binding energy ( $\left.E_{b i n d i n g}\right)$ for 5 OPFRs.

\begin{tabular}{|c|c|c|c|c|c|c|}
\hline No. & Compound & Abb. & $k_{a}(1 / \mathrm{Ms})$ & $k_{d}(1 / \mathrm{s})$ & $K_{D}(\mathrm{M})$ & $E_{\text {binding }}(\mathrm{kJ} / \mathrm{mol})$ \\
\hline 1 & Tris(2-chloroethyl)phosphate & TCEP & $5.19 \times 10^{3}$ & $1.45 \times 10^{-2}$ & $2.79 \times 10^{-6}$ & -94.33 \\
\hline 2 & Tri- $n$-propylphosphate & TPrP & $1.44 \times 10^{4}$ & $4.07 \times 10^{-2}$ & $2.82 \times 10^{-6}$ & -87.40 \\
\hline 3 & Di-n-butylphosphate & DnBP & $1.16 \times 10^{3}$ & $4.39 \times 10^{-2}$ & $3.8 \times 10^{-5}$ & -18.24 \\
\hline 4 & Triethylphosphate & TEP & $6.11 \times 10^{3}$ & $7.29 \times 10^{-2}$ & $1.19 \times 10^{-5}$ & -7.20 \\
\hline 5 & Tributylphosphate & TnBP & $5.22 \times 10^{3}$ & $4.16 \times 10^{-2}$ & $7.97 \times 10^{-6}$ & -62.42 \\
\hline
\end{tabular}
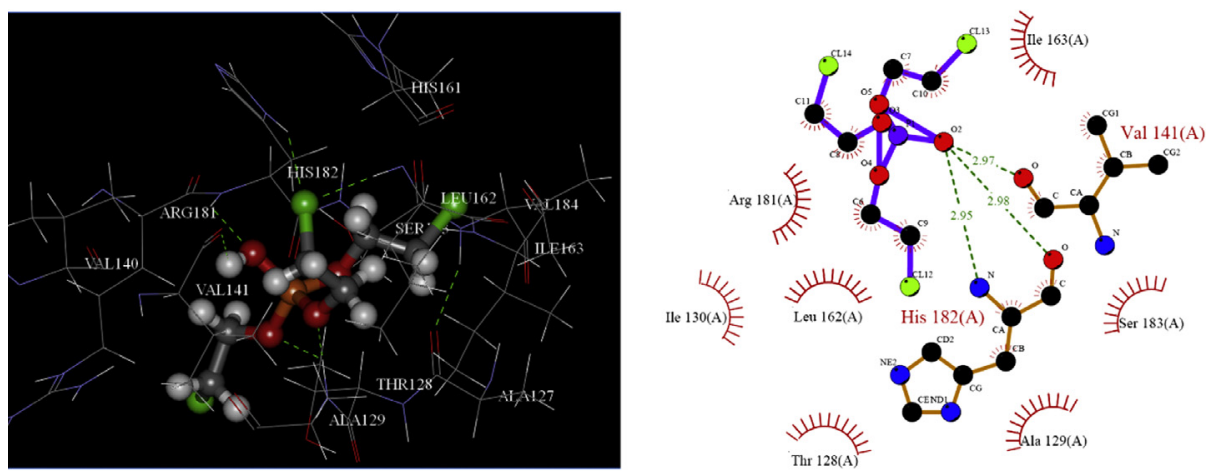

(a) Tris(2-chloroethyl)phosphate
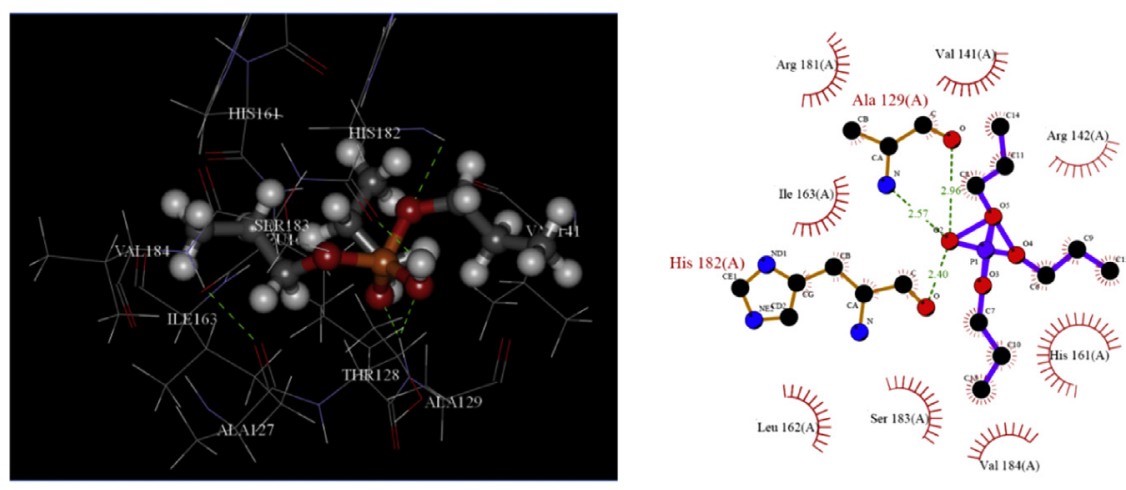

(b) Tri-n-propylphosphate
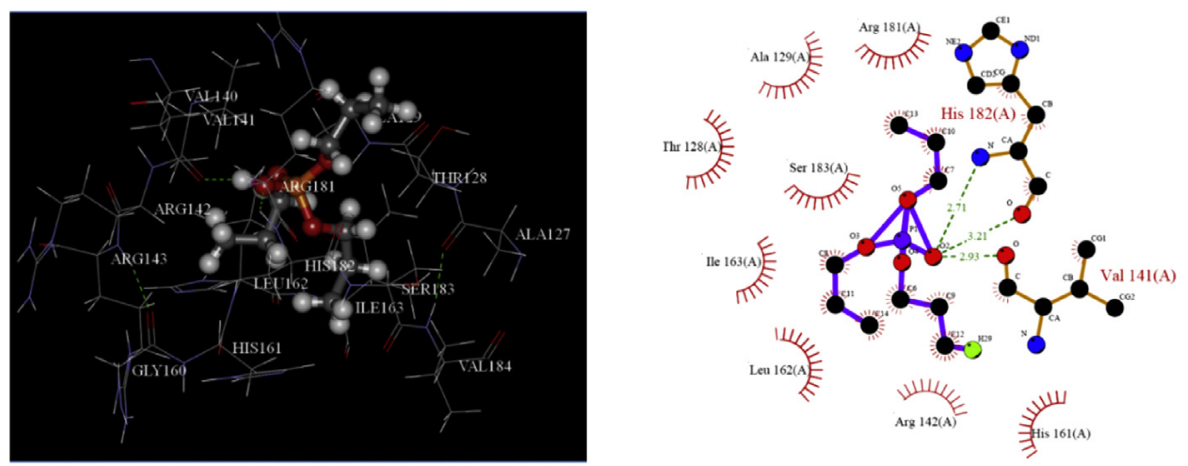

(c) Tri-n-propylphosphate
$\longrightarrow$ ligand bond
$\longrightarrow$ receptor bond
- Hydrogen bond
receptor residues involved
in hydrophobic interactions
- Corresponding atoms involved in hydrophobic interactions

Fig. 2. Hydrogen bondings (left) and hydrophobic interactions (right) between TCEP, TEP and TPrP in the binding site of p53. 


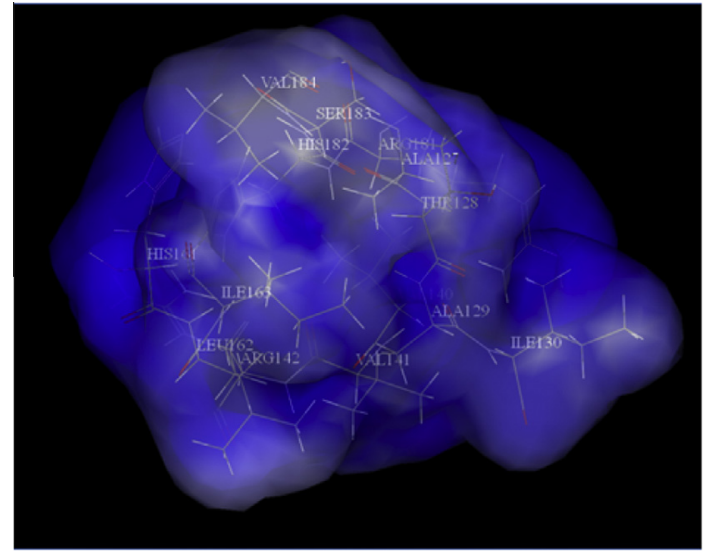

Fig. 3. Electrostatic potential of the ligand binding site for p53.

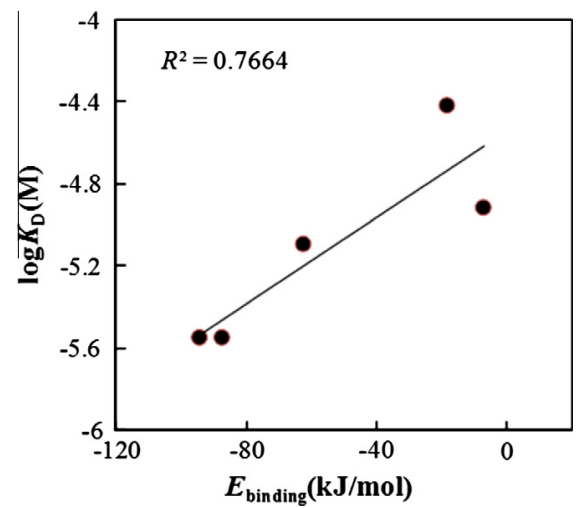

Fig. 4. Plot of calculated $E_{\text {binding }}$ values $v s$, the determined $\log K_{D}$. $E_{\text {binding }}$ : receptor binding energy $(\mathrm{kJ} / \mathrm{mol}) ; K_{D}$ : the binding constant.

involvement of $E_{\text {binding }}$ in the QSAR model implies the effects of ligand-receptor interaction on the estrogenic activity.

\section{Conclusions}

The binding constants of 5 OPFRs were determined by surface plasmon resonance (SPR). Docking analysis showed that hydrogen bonding and hydrophobic interactions between the ligand molecules and p53 governed the binding activities of the OPFRs. Based on the mechanism of action, a QSAR was developed to characterize the interactions and model the binding affinities of the OPFRs. The partition ability of the OPFRs into the bio-phase was significant parameters related to the binding interaction. The developed QSAR model has good robustness, predictive ability, and mechanism interpretability.

\section{Acknowledgments}

This research was supported by the National Natural Science Foundation of China (21107136), the International Foundation for Science (F/5230-1), and the Key Laboratory of Industrial Ecology and Environmental Engineering and China Ministry of Education (KLIEEE-10-09).

\section{References}

Andresen, J., Bester, K., 2006. Elimination of organophosphate ester flame retardants and plasticizers in drinking water purification. Water Res. 40, 621-629.
Andresen, J.A., Grundmann, A., Bester, K., 2004. Organophosphorus flame retardants and plasticisers in surface waters. Sci. Total Environ. 332, $155-166$.

Bacaloni, A., Cucci, F., Guarino, C., Nazzari, M., Samperi, R., Lagana, A., 2008. Occurrence of organophosphorus flame retardant and plasticizers in three volcanic lakes of Central Italy. Environ. Sci. Technol. 42, 1898-1903.

Campbell, K., Huet, A.C., Charlier, C., Higgins, C., Delahaut, P., Elliott, C.T., 2009. Comparison of ELISA and SPR biosensor technology for the detection of paralytic shellfish poisoning toxins. J. Chromatogr. B 877, 4079-4089.

Commission, E., 2001. European Chemicals Bureau. IUCLID, dataset Tris(2chloromethyl) phosphate, EINECS No. 204-118-5; <http://ecb.jrc.it/ESIS>.

David, M.D., Seiber, J.N., 1999. Analysis of organophosphate hydraulic fluids in US Air Force base soils. Arch. Environ. Contam. Toxicol. 36, 235-241.

Dishaw, L.V., Powers, C.M., Ryde, I.T., Roberts, S.C., Seidler, F.J., Slotkin, T.A., Stapleton, H.M., 2011. Is the PentaBDE replacement, tris (1,3-dichloropropyl) phosphate (TDCPP), a developmental neurotoxicant? Studies in PC12 cells. Toxicol. Appl. Pharmacol. 256, 281-289.

Drake, A.W., Tang, M.L., Papalia, G.A., Landes, G., Haak-Frendscho, M., Klakamp, S.L., 2012. Biacore surface matrix effects on the binding kinetics and affinity of an antigen/antibody complex. Anal. Biochem. 429, 58-69.

Garcia-Lopez, M., Rodriguez, I., Cela, R., 2009. Pressurized liquid extraction of organophosphate triesters from sediment samples using aqueous solutions. J. Chromatogr. A 1216, 6986-6993.

Hartmann, P.C., Burgi, D., Giger, W., 2004. Organophosphate flame retardants and plasticizers in indoor air. Chemosphere 57, 781-787.

Karlsson, R., 2004. SPR for molecular interaction analysis: a review of emerging application areas. J. Mol. Recognit. 17, 151-161.

Katayama, M., Sato, T., Kuromitsu, J., 2012. Capture molecules preconditioned for kinetic analysis of high-affinity antigen-antibody complex in Biacore A100. Anal. Biochem. 424, 168-177.

Li, F., Li, X.H., Shao, J.P., Chi, P., Chen, J.W., Wang, Z.J., 2010a. Estrogenic activity of anthraquinone derivatives: in vitro and in silico studies. Chem. Res. Toxicol. 23, $1349-1355$.

Li, F., Xie, Q., Li, X.H., Li, N., Chi, P., Chen, J.W., Wang, Z.J., Hao, C., 2010b. Hormone activity of hydroxylated polybrominated diphenyl ethers on human thyroid receptor $\beta$ : in vitro and in silico investigations. Environ. Health Perspect. 118, 602-606.

Li, F., Wu, H.F., Li, L.Z., Li, X.H., Zhao, J.M., Peijnenburg, W.J.G.M., 2012. Docking and QSAR study on the binding interactions between polycyclic aromatic hydrocarbons and estrogen receptor. Ecotox. Environ. Safe. 80, 273-279.

Liu, X., Ji, K., Choi, K., 2012. Endocrine disruption potentials of organophosphate flame retardants and related mechanisms in H295R and MVLN cell lines and in zebrafish. Aquat Toxicol. 114, 173-181.

Marklund, A., Andersson, B., Haglund, P., 2003. Screening of organophosphorus compounds and their distribution in various indoor environments. Chemosphere 53, 1137-1146.

Martinez, L., Polikarpov, I., Skaf, M.S., 2008. Only subtle protein conformational adaptations are required for ligand binding to thyroid hormone receptors: simulations using a novel multipoint steered molecular dynamics approach. J. Phys. Chem. B 112, 10741-10751.

Martinez-Carballo, E., Gonzalez-Barreiro, C., Sitka, A., Scharf, S., Gans, O., 2007. Determination of selected organophosphate esters in the aquatic environment of Austria. Sci. Total Environ. 388, 290-299.

Matthews, H.B., Dixon, D., Herr, D.W., Tilson, H., 1990. Subchronic toxicity studies indicate that tris(2-chloroethyl)phosphate administration results in lesions in the rat hippocampus. Toxicol. Ind. Health 6, 1-15.

Meyer, J., Bester, K., 2004. Organophosphate flame retardants and plasticisers in wastewater treatment plants. J. Environ. Monit. 6, 599-605.

OECD, 2007. Guidance document on the validation of (Quantitative) StructureActivity Relationships [(Q)SARs] models. Available online at: <http:// appli1.oecd.org/olis/2007doc.nsf/linkto/env-jm-mono(2007)2>.

Reemtsma, T., Quintana, J.B., Rodil, R., Garcia-Lopez, M., Rodriguez, I., 2008 Organophosphorus flame retardants and plasticizers in water and air I. Occurrence and fate. TrAC-Trends in Anal. Chem. 27, 727-737.

Regnery, J., Puttmann, W., 2010. Occurrence and fate of organophosphorus flame retardants and plasticizers in urban and remote surface waters in Germany. Water Res. 44, 4097-4104.

Schüürman, G., Ebert, R.U., Chen, J.W., Wang, B., Kuhne, R., 2008. External validation and prediction employing the predictive squared correlation coefficient - test set activity mean vs. training set activity mean. J. Chem. Inf. Model. 48, 2140 2145 .

Sipova, H., Homola, J., 2013. Surface plasmon resonance sensing of nucleic acids: a review. Anal. Chim. Acta 773, 9-23.

Stackelberg, P.E., Furlong, E.T., Meyer, M.T., Zaugg, S.D., Henderson, A.K., Reissman, D.B., 2004. Persistence of pharmaceutical compounds and other organic wastewater contaminants in a conventional drinking-watertreatment plant. Sci. Total Environ. 329, 99-113.

Stapleton, H.M., Klosterhaus, S., Eagle, S., Fuh, J., Meeker, J.D., Blum, A., Webster, T.F. 2009. Detection of organophosphate flame retardants in furniture foam and US house dust. Environ. Sci. Technol. 43, 7490-7495.

Takigami, H., Suzuki, G., Hirai, Y., Ishikawa, Y., Sunami, M., Sakai, S., 2009. Flame retardants in indoor dust and air of a hotel in Japan. Environ. Int. 35 688-693. 
Wu, G.S., Robertson, D.H., Brooks, C.L., Vieth, M., 2003. Detailed analysis of gridbased molecular docking: A case study of CDOCKER - A CHARMm-based MD docking algorithm. J. Comput. Chem. 24, 1549-1562.

Xue, Y.L., Wang, S.A., Feng, X.Z., 2009. Effect of metal ion on the structural stability of tumour suppressor protein p53 DNA-binding domain. J. Biochem. 146, 193200.
Yasuhara, A., Shiraishi, H., Nishikawa, M., Yamamoto, T., Nakasugi, O., Okumura, T., Kenmotsu, K., Fukui, H., Nagase, M., Kawagoshi, Y., 1999. Organic components in leachates from hazardous waste disposal sites. Waste Manage. Res. 17, 186197. 